\title{
Frailty is to predictive as Jello is to wall
}

\author{
Craig R. Smith, MD
}

\footnotetext{
From the Department of Surgery, Columbia University Medical Center of New York Presbyterian Hospital, New York, NY.

Disclosures: Author has nothing to disclose with regard to commercial support.

Received for publication Jan 29, 2018; accepted for publication Jan 31, 2018; available ahead of print Feb 28, 2018.

Address for reprints: Craig R. Smith, MD, Department of Surgery, Columbia University Medical Center of New York Presbyterian Hospital, Milstein Building 7-435 177 Fort Washington Ave, New York, NY 10032 (E-mail: crs2@columbia.edu).

J Thorac Cardiovasc Surg 2018;156:177

$0022-5223 / \$ 36.00$

Copyright (C) 2018 by The American Association for Thoracic Surgery

https://doi.org/10.1016/j.jtcvs.2018.01.068
}

Back in the unenlightened era in which I trained, we talked about patients having "poor protoplasm," a quality we eyeballed with our "protoplasmometer," and superstitiously embraced as an uncanny predictor of bad outcomes. Such cynicisms have no place today! Protoplasm deserves as much respect as any mitochondrion or nucleolus. Thankfully, we now have "frailty" to characterize the outcomepredicting essence we were attempting to capture. And the definition of "frailty" is....

This is precisely our dilemma, and that of Yanagawa and colleagues, ${ }^{1}$ who have reviewed the topic of frailty as a cardiac surgery risk predictor with the goal of relegating the eyeball test to medical history. The authors don't consider why interest in frailty has exploded in the past decadecertainly one reason is that interventional cardiologists realized how effectively the "frail" label could be used to move patients from surgery to transcatheter aortic valve replacement. The authors discuss the roles that frailty could play in risk prediction algorithms and in setting up targets for "de-frailing" through preoperative physical rehabilitation, metabolic optimization, and similar strategies. However, those laudable benefits depend entirely on our ability to develop a meaningful definition of frailty that can be shown to have predictive value. Fried's pioneering "phenotypic" approach aggregates variables (eg, strength, gait, daily activities) thought to define a true "biological syndrome" that excludes comorbidities (eg, renal dysfunction, chronic obstructive pulmonary disease) ${ }^{2}$ At the other extreme are "index-based tools," such as the Rockwood Frailty Index, that can generate a continuous variable out of many individual features. By now, the literature includes many claims for predictability of many different outcomes using many different frailty definitions assembled from a vast menu of options. There are significant practical challenges-for either the phenotypic or index-based approach, who will perform the testing, who will record and analyze as many futility.

\section{References} 2018;156:172-6.e2.

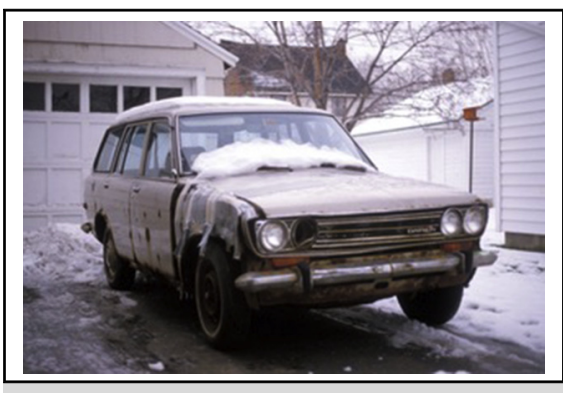

1970 Datsun 510 duct tape fenderoplasty.

Central Message

This thorough review of current definitions and implications of frailty demonstrates that although much progress has been made, a simple, reproducible, and highly predictive definition of frailty remains illusory.

See Article page 172.

as 92 variables for an index, and who will ensure that definitions and testing standards for each variable are uniform? As the authors note, the Society of Thoracic Surgeons database has recently dipped a toe in the frailty waters by adding one reasonably simple and reproducible metric, the 5-meter walk. A single variable like the 5-meter walk is probably somewhat predictive, but will it be better than, or even as good as, one good pair of eyeballs? Frailty may be the bullseye that's defined by all the darts that miss it.

There is a somewhat Calvinist quandary at the root of the "frail" label. Is frailty reversible? Or is irreversibility the essence of frailty? Many of the factors used to define frailty are reversible, yet we've all seen operations or other interventions that improve the function of various organs without keeping the patient out of a nursing home. Frailty may be the unimprovable residue of age and debilitating diseases that merges with another very important f-word:

1. Yanagawa B, Graham MM, Afilalo J, Hassan A, Arora RC. Frailty as a risk predictor in cardiac surgery: beyond the eyeball test. J Thorac Cardiovasc Surg.

2. Fried LP, Tangen CM, Walston J, Williamson JD, Anderson G. Untangling the concepts of disability, frailty, and comorbidity: implications for improved targeting and care. J Gerontol A Biol Sci Med Sci. 2001;56:M146-56. 